\title{
Subprodutos do babaçu (Orbignya sp) como novos materiais adsortivos: uma revisão
}

\section{Byproducts of babassu (Orbignya sp) as new adsorptive materials: a review}

\author{
Josany Saibrosa da Silva ${ }^{1}$, Mirian Lima dos Santos ${ }^{2}$, \\ Edson Cavalcante da Silva Filho ${ }^{1}$, Maria das Graças Freire de Medeiros Carvalho ${ }^{3}$, \\ Lívio César Cunha Nunes ${ }^{3}$
}

\footnotetext{
${ }^{1}$ Programa de Pós-Graduação em Ciência dos Materiais- PPGCM/ UFPI, CEP: 64049-550, Teresina, PI, Brasil.

${ }^{2}$ Programa Institucional de Bolsa de Iniciação Científica- PIBIC UFPI, CEP: 64049-550, Teresina, PI, Brasil.

${ }^{3}$ Programa de Pós-Graduação em Ciências Farmacêuticas- PPGCF UFPI, CEP: 64049-550, Teresina, PI, Brasil. e-mail: josany.saibrosa@gmail.com, mir_ianbr@hotmail.com,mgfmedeiros@hotmail.com, edsonfilho@ufpi.edu.br liviocesar@hotmail.com
}

\begin{abstract}
RESUMO
O Babaçu (Orbignya sp) é uma planta lignocelulósica, disponível em larga escala no norte-nordeste do Brasil, que vem despontado como uma boa fonte de biomateriais, sendo utilizados em vários estudos como adsorventes. Diante disso, o presente trabalho teve como objetivo realizar um estudo prospectivo do uso de subprodutos do babaçu (endocarpo, mesocarpo, epicarpo e carvão ativado) como um material com excelentes propriedades adsortivas, em potencial, para moléculas inorgânicas e orgânicas.O estudo foi realizado através de uma revisão de literatura (artigos e patentes), no intervalo temporal de julho de 2015 a fevereiro de 2018. As bases de dados utilizadas foram: Instituto Nacional de Propriedade Industrial do Brasil, World Intellectual Property Organization, European Patent Office, United States Patent and Trademark Office e Patentes públicas da América latina e Espanha, para busca de patentes; e Pubmed, Scopus, Web of Science e Scientific Electronic Library Online, para os artigos. A partir da análise criteriosa do levantamento bibliográfico foram selecionados 18 artigos científicos e 1 patente, sobre o tema. Dentre os quais o mesocarpo, epicarpo (naturais e modificados) e o carvão ativado do babaçu foram substratos de adsorção bastante eficientes. Onde os resultados foram satisfatórios, de modo que o babaçu desponta como um bom adsorvente e de baixo custo, sendo assim um biomaterial de qualidade e acessível.
\end{abstract}

Palavras-chave: Adsorção, Babaçu, Descontaminação, Meio ambiente.

\begin{abstract}
Babaçu (Orbignya sp) is a lignocellulosic plant, available in large scale in the north-northeast of Brazil, which has been emerging as a good source of biomaterials, being used in several studies as adsorbents. The objective of this study was to prospectively study the use of babaçu by-products (endocarp, mesocarp, epicarp and activated carbon) as a material with the exception of potential adsorptive target species for inorganic and organic molecules. was carried out through a literature review (article and patents), in the time period of July 2015 and February 2018. The databases used were: National Institute of Industrial Property of Brazil, World Intellectual Property Organization, Patents, Patent and Trademark Office of the United States of America and Spain for Patent Search; and Pubmed, Scopus, Web of Science and Scientific Electronic Library Online, for articles. From the careful analysis of the bibliographical survey, 18 scientific papers and 1 patent on the subject were selected. Among them, the mesocarp, epicarp (natural and modified) and the activated charcoal of babassu were very efficient adsorption substrates. The results were satisfactory, so that babassu was shown as a good indicator of cost and benefit, thus being a quality and accessible biomaterial.
\end{abstract}

Keywords: Adsorption, babassu, Decontamination, Environment. 


\section{INTRODUÇÃO}

O Desenvolvimento econômico e industrial trouxeram consigo uma série de benefícios, mas também trouxeram consequências. A contaminação de solos e águas depois da industrialização apenas aumentou, causando desequilíbrio ecológico em ecossistemas naturais ainda não alterados por ação antrópica. E acarretando a geração de diversos problemas ambientais, sociais e de saúde, sendo a indústria química uma das maiores responsáveis por este processo[1,2].

As técnicas de adsorção são amplamente usadas para remover certas classes de poluentes das águas, especialmente aqueles que não são facilmente biodegradáveis ou possuem moléculas muito pequenas. Numerosos estudos têm sido desenvolvidos a fim de investigar a utilização de adsorventes alternativos, sejam materiais orgânicos ou inorgânicos. Estes materiais estão disponíveis em grandes quantidades na natureza, como materiais naturais de origem mineral, vegetal ou animal [3-5].

O babaçu é uma planta nativa do Brasil, disseminada por quase todo o interior do Brasil, desde o estado do Amazonas até o estado de São Paulo. Contudo, são nos estados do Maranhão, Piauí, e algumas áreas isoladas no Ceará, Pernambuco e Alagoas, onde se localizam as principais ocorrências dessa palmeira. Tendo o fruto composto por partes distintas (Figura 1). O Epicarpo é a camada externa do fruto do babaçu, possui estrutura fibrosa, corresponde a $12 \%$ do fruto e apresenta cor amarelo-avermelhada. O Mesocarpo é camada abaixo do epicarpo, corresponde a $23 \%$ do fruto, tem aspecto farinhoso e é rica em amido. Dependendo do grau de maturação do fruto, apresenta cor branco-amarelada, é a camada intermediária, com espessura de até $1 \mathrm{~cm}$. O Endocarpo protege as amêndoas, é de onde se produz um carvão vegetal com alta qualidade, corresponde a $58 \%$ do fruto. Em geral, possui coloração marrom. A parte central do fruto do babaçu é composta por sementes onde cada fruto possui de três a quatro amêndoas, das quais se extrai o óleo vegetal, $7 \%$ do fruto [6].

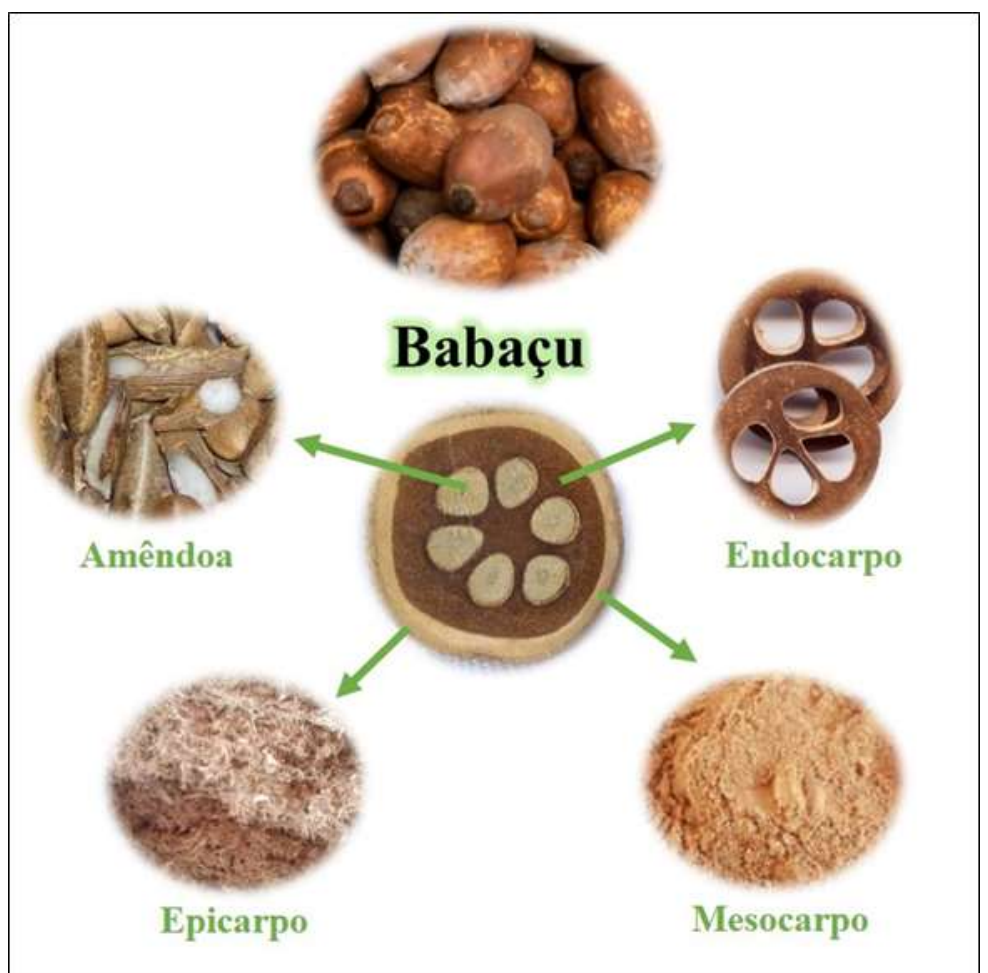

Figura 1: Coco babaçu e seus componentes. Fonte: autoria própria (2018)

A sua área de ocorrência natural no Brasil é avaliada em cerca de 18 milhões de hectares. Somente a região Nordeste possui uma área de cerca de 14 milhões de hectares com babaçu, sendo que a maior parte (54,2\%) concentra-se no Estado do Maranhão [7]. Essa grande abundância do coco babaçu juntamente com suas características físico-químicas o tornam um material de diversas utilidades, inclusive sendo utilizado como material adsortivo de moléculas químicas. Essa propriedade adsortiva dos subprodutos do babaçu (en- 
docarpo, epicarpo, mesocarpo, carvões, entre outros), se deve, principalmente, pela característica lignocelulósica dos seus constituintes e pela presença (ou formação) de superfícies mesoporosas [8].

Com isso, o babaçu desponta hoje com um grande potencial em vários âmbitos tecnológicos [9]. E diante disso, o presente trabalho teve como objetivo realizar um estudo prospectivo científico e tecnológico do uso de subprodutos do babaçu como materiais adsortivos para moléculas químicas e, consequentemente, como materiais descontaminantes do meio ambiente.

\section{MATERIAIS E MÉTODOS}

A prospecção científica e tecnológica foi realizada com base nos pedidos de patentes e de artigos científicos publicados entre 2015 e 2018. As bases de dados utilizadas foram: Instituto Nacional de Propriedade Industrial do Brasil (INPI), World Intellectual Property Organization (WIPO), European Patent Office (EPO), United States Patent and Trademark Office (USPTO) e Patentes públicas da América latina e Espanha (Latipat), para busca de patentes; e Pubmed, Scopus, Web of Science e Scientific Electronic Library Online (Scielo), para os artigos. As palavras-chave empregadas foram "babaçu", "adsorvente", "adsorção" e "sorção" (juntas e separadas), em português e inglês. Sendo válidos os resultados que apresentassem os termos descritos nos campos de pesquisa título e resumo, sem exclusão de trabalhos por ano de publicação até a data da pesquisa.

\section{RESULTADOS}

O uso de materiais adsorventes provenientes de biomassa é atraente devido a sua participação na redução de custos para eliminação de resíduos. Por isso, vários pesquisadores têm buscado materiais de baixo custo para viabilizar novos adsorventes [10]. De acordo com levantamentos dos trabalhos já publicados, o babaçu é um adsorvente em potencial, tendo nele acumulado um baixo custo de produção e alta disponibilidade.

A pesquisa teve com resultado a Tabela 1. Após exclusão de artigos em duplicata ou triplicata e sobre temas não referentes à descontaminação do meio ambiente, apenas 18 artigos foram integralizados nesta revisão. Descritos na Tabela 2.

Tabela 1: Palavras-chaves utilizadas e números de artigos encontrados na pesquisa.

\begin{tabular}{|c|c|c|c|c|}
\hline PALAVRAS-CHAVES & WEB OF SCIENCE & PUBMED & SCOPUS & SCIELO \\
\hline $\begin{array}{l}\text { Babaçu e Adsorção/ babassu and ad- } \\
\text { sorption }\end{array}$ & 27 & 8 & 26 & 8 \\
\hline $\begin{array}{l}\text { Babaçu e adsorvente/ babassu and } \\
\text { adsorbent }\end{array}$ & 12 & 1 & 9 & 2 \\
\hline Babaçu e sorção/ babassu and sorption & 11 & 1 & 9 & 3 \\
\hline Total & \multicolumn{4}{|c|}{117 artigos } \\
\hline
\end{tabular}

Tabela 2: Estudos que utilizam o babaçu como material adsorvente para remoção de contaminantes no meio ambiente.

\begin{tabular}{|c|c|c|c|c|}
\hline MATERIAL ADSORVIDO & MEIO & $\begin{array}{c}\text { SUBPRODUTO DO } \\
\text { BABAÇU }\end{array}$ & $\begin{array}{l}\text { ADSORÇÃO/ RE- } \\
\text { MOÇÃO DO MEIO }\end{array}$ & REFERÊNCIA \\
\hline Cloro residual & Solução aquosa & Carvão ativado & $40 \%$ após $60 \mathrm{~min}$ & [11] \\
\hline Prata & $\begin{array}{l}\text { Águas residuais } \\
\text { radiográficas }\end{array}$ & Carvão ativado & $\begin{array}{c}0,42 \mathrm{mg} / \mathrm{g} \text { de carvão } \\
\text { ativado }\end{array}$ & [12] \\
\hline \multirow[t]{4}{*}{ Corante (Azul reativo $5 \mathrm{G}$ ) } & Solução aquosa & Carvão ativado & $\begin{array}{c}0,77 \mathrm{mg} / \mathrm{g} \text { de carvão } \\
\text { ativado }\end{array}$ & [13] \\
\hline & & & $\begin{array}{c}12,9 \text { mg/ g de carvão } \\
\text { ativado }\end{array}$ & [14] \\
\hline & & Carvão ativado & $97 \%$ & [15] \\
\hline & & $\begin{array}{l}\text { Carvão ativado modi- } \\
\text { ficado com ácido cítri- }\end{array}$ & $1,54 \mathrm{mg} / \mathrm{g}$ & [16] \\
\hline Corante (Azul metileno) & Solução aquosa & co & & \\
\hline
\end{tabular}




\begin{tabular}{|c|c|c|c|c|}
\hline & & Mesocarpo & Análise visual & [17] \\
\hline Cafeína & Solução aquosa & Carvão ativado & $\begin{array}{c}250 \text { mg/ g de carvão } \\
\text { ativado }\end{array}$ & [18] \\
\hline Albumina de soro bovino & Solução aquosa & Carvão ativado & $36,18 \mathrm{mg} / \mathrm{g}$ de carvão & [19] \\
\hline Nitrato & Extrato aquoso & \multirow{2}{*}{$\begin{array}{l}\text { Carvão ativado im- } \\
\text { pregnado com cobre }\end{array}$} & $\begin{array}{c}45,1 \mathrm{mg} / \mathrm{g} \text { de carvão } \\
\text { ativado }\end{array}$ & [20] \\
\hline $\begin{array}{c}\text { Compostos sulfúricos e } \\
\text { nitrogenados (derivados de } \\
\text { diesel) }\end{array}$ & Diesel & & $\begin{array}{c}64,7 \% \text { de compostos } \\
\text { sulfúricos e } 83,3 \% \text { de } \\
\text { compostos nitroge- } \\
\text { nados }\end{array}$ & [21] \\
\hline Corante (Azul remazol & Solução aquosa & Mesocarpo & Entre 43,4 a $99,5 \%$ & [22] \\
\hline $\begin{array}{l}\text { R160, Rubi S2G, Vermrlho } \\
\text { remazol 5R, Violeta remazol } \\
\text { 5R e Verde oliva indatreno) }\end{array}$ & Solução aquosa & Epicarpo & $\begin{array}{c}\text { Entre } 0,3 \text { e } 3,0 \text { mg/ g } \\
\text { de epicapo }\end{array}$ & {$[8]$} \\
\hline Corante (Turquesa remazol) & Solução aquosa & Mesocarpo e epicarpo & $\begin{array}{l}1,44 \text { e } 2,38 \mathrm{mg} / \mathrm{g} \text { de } \\
\text { mesocarpo e epicar- } \\
\text { po, respectivamente }\end{array}$ & [10] \\
\hline Íons metálicos & Solução aquosa & $\begin{array}{l}\text { Mesocarpo e epicarpo } \\
\text { modificados (etileno- } \\
\text { sulfidríco) }\end{array}$ & $\begin{array}{l}39,6 \text { e } 39,2 \mathrm{mg} / \mathrm{g} \text { de } \\
\text { mesocarpo e epicar- } \\
\text { po modificados, res- } \\
\text { pectivamente }\end{array}$ & [23] \\
\hline Cobre & Cachaça & $\begin{array}{l}\text { Mesocarpo modifica- } \\
\text { do (anidrido succínico, } \\
\text { maleico ou ftálico) }\end{array}$ & $\begin{array}{c}\text { Redução de } 1 \mathrm{~g} / \mathrm{dm}^{3} \\
\text { para } 5 \mathrm{mg} / \mathrm{dm}^{3}\end{array}$ & [24] \\
\hline Cobre & Solução aquosa & $\begin{array}{l}\text { Epicarpo (anidrido } \\
\text { maleico) }\end{array}$ & $\begin{array}{c}55,09 \mathrm{mg} / \mathrm{g} \text { de epi- } \\
\text { carpo }\end{array}$ & [25] \\
\hline $\begin{array}{c}\text { Dodecilbenzenossulfonato } \\
\text { de Sódio }\end{array}$ & Solução aquosa & $\begin{array}{l}\text { Farinha fresca de } \\
\text { mesocarpo }\end{array}$ & $6,5 \%$ & [26] \\
\hline
\end{tabular}

Fonte: autoria própria (2018)

E, em relação às patentes encontradas, o resultado foi de apenas 2 patentes (Tabela 3), sendo que uma não se enquadrou no quesito descontaminação do meio ambiente, já que se tratava apenas do uso do óleo de babaçu em produtos cosméticos, cujo número de depósito o é JPS62192308 (A), com data de deposito 22/08/1987. Restando, assim, apenas uma patente do uso de um subproduto de babaçu como material adsorvente de compostos químicos [27].

Tabela 3: Palavras-chaves utilizadas na pesquisa de patentes.

\begin{tabular}{lccccc}
\hline PALAVRAS-CHAVES & INPI & ESPACENET & WIPO & USPTO & LATIPAT \\
\hline $\begin{array}{l}\text { Babaçu e Adsorção/ babassu } \\
\text { and adsorption }\end{array}$ & 0 & 1 & 0 & 0 & 0 \\
\hline $\begin{array}{l}\text { Babaçu e adsorvente/ babassu } \\
\text { and adsorbent }\end{array}$ & 1 & 0 & 0 & 0 & 0 \\
\hline $\begin{array}{l}\text { Babaçu e sorção/ babassu and } \\
\text { sorption }\end{array}$ & 0 & 0 & 0 & 0 & 0 \\
\hline$\quad$ Total & & 2 patentes & \\
\hline
\end{tabular}

Fonte: autoria própria (2018)

A patente encontrada, cujo número de depósito é PI 100448-7 A2, com data de depósito em 29/07/2010, tem como inovação a obtenção e o uso de Mesocarpo modificado com anidrido succínico, ftálico 
e maleico para remoção de íons metálicos em soluções hidroetanólicas e em meio aquoso [28].

Observa-se que pesquisas vêm sendo realizadas a nível nacional já que o coco babaçu, é nativo brasileiro, no entanto, publicadas em periódios internacionais. Atitudes como essas de divulgar cada vez mais trabalhos relacionados ao tema pode levar novos pesquisadores a conhecer o poder de adsorção dos subprodutos do babaçu. De forma que novos estudos poderão ser realizados e reconhecidos a nível mundial [29].

\section{DISCUSSÃO}

\subsection{O processo de adsorção}

A adsorção é um importante método para a remoção de moléculas a partir de meios aquosos. É um fenômeno de superfície, o qual está relacionado com a área disponível do adsorvente em relação às massas do adsorbato e do adsorvente, $\mathrm{pH}$, temperatura, força iônica e a natureza química do adsorvente e adsorbato, podendo ser reversível ou irreversível. As moléculas que estão presentes em um fluido, líquido ou gasoso, concentram-se espontaneamente sobre uma superfície sólida. Geralmente, a adsorção parece ocorrer como um resultado de forças não balanceadas na superfície do sólido e que atraem as moléculas de um fluido em contato por um tempo finito. Este processo encontra grande aplicação industrial, pois associa baixo custo, elevadas taxas de remoção, facilidade de operação e insensibilidade a substâncias tóxicas [30, 31].

A relação entre a quantidade de material adsorvido na superfície do adsorvente e a concentração de adsorbato remanescente no equilíbrio sólido/líquido é prevista por meio das isotermas de adsorção. Dentre as quais pode-se destacar os modelos matemáticos de Langmuir, Freundlich, Temkin e Dubinin-Radushkevich. O modelo de Langmuir pressupõe que os locais de ligação são homogeneamente distribuídos sobre a superfície do adsorvente com afinidade de adsorção de modo a formar uma única camada molecular. Enquanto que o modelo de Freundlich descreve a multicamada de adsorção com a interação entre as moléculas adsorvidas e também prevê que as concentrações de adsorbato sobre o material aumentará com um aumento da concentração do mesmo na solução, relacionado com uma distribuição uniforme de energia de ligação. O modelo de Temkin também é caracterizado por uma distribuição uniforme de energia de ligação, mas apenas até uma energia de ligação máxima. E o modelo de Dubinin-Radushkevich expressa uma constante que dá a energia livre média de adsorção por molécula de adsorbato que é transferido para a superfície do sólido (adsorvente) em solução [14,22].

Modelos cinéticos tem sido desenvolvidos para investigar os mecanismos de adsorção e as etapas controladoras do processo, incluindo a transferência de massa e as reações químicas, se for o caso. Os modelos mais empregados são o de pseudo-primeira ordem, pseudo-segunda ordem e difusão intrapartícula.Relacionados, respectivamente, à capacidade de adsorção do sólido; ao comportamento do processo em toda a faixa de tempo de contato; e à difusão intrapartícula das moléculas no adsorvente [16, 22].

A técnica de adsorção oferece mais flexibilidade e é vantajosa em relação a outras técnicas, tais como precipitação, permuta iônica, floculação e electrodeposição, porque, em muitos casos, o efluente tratado pode ser reutilizado [32]. O processo de adsorção é muitas vezes reversível, de modo que a modificação da temperatura e/ou pressão, potencial hidrogeniônico pode provocar a fácil remoção do soluto adsorvido no sólido. Isso se torna mais uma vantagem desse método, já que moléculas podem ser, de certa forma, reaproveitadas e reutilizadas a partir de um sistema adsorvente eficiente. Além de ser uma metodologia que auxilia na descontaminação do meio ambiente, também pode diminuir custos e continuar ajudando o meio ambiente com a diminuição de produção de moléculas químicas, como os corantes e metais pesados [32, 33].

\subsection{Babaçu como adsorvente}

O babaçu é um biopolímero, constituído principalmente por fibras lignocelulósicas [8,25]. E devido às ligações inter e intramoleculares que a liginina e a celulose são capazes de realizar, suas fibras tem capacidade adsortiva [34].

Os subprodutos do babaçu vêm se mostrando como materiais altamente eficientes e interessantes para serem utilizados na adsorção de corantes [8, 10, 13-15, 22], íons [10-12, 23-25], entre outras moléculas químicas [18,35]. Percebe-se, portanto, que as substâncias já adsorvidas em babaçu são moléculas pequenas e de difícil remoção por outros métodos. Com o maior número de estudos para remoção de corantes de águas residuais (Figura 2), os quais são um dos principais constituintes dos efluentes produzidos a partir de muitas indústrias relacionadas com a matéria têxtil, tintas e vernizes, plásticos, curtume, etc. Os corantes representam uma grande ameaça para o ecossistema circundante[13]. Assim como a presença de íons metálicos em água residuais, tóxicos ao meio ambiente e, principalmente, aos humanos e animais [12]. 


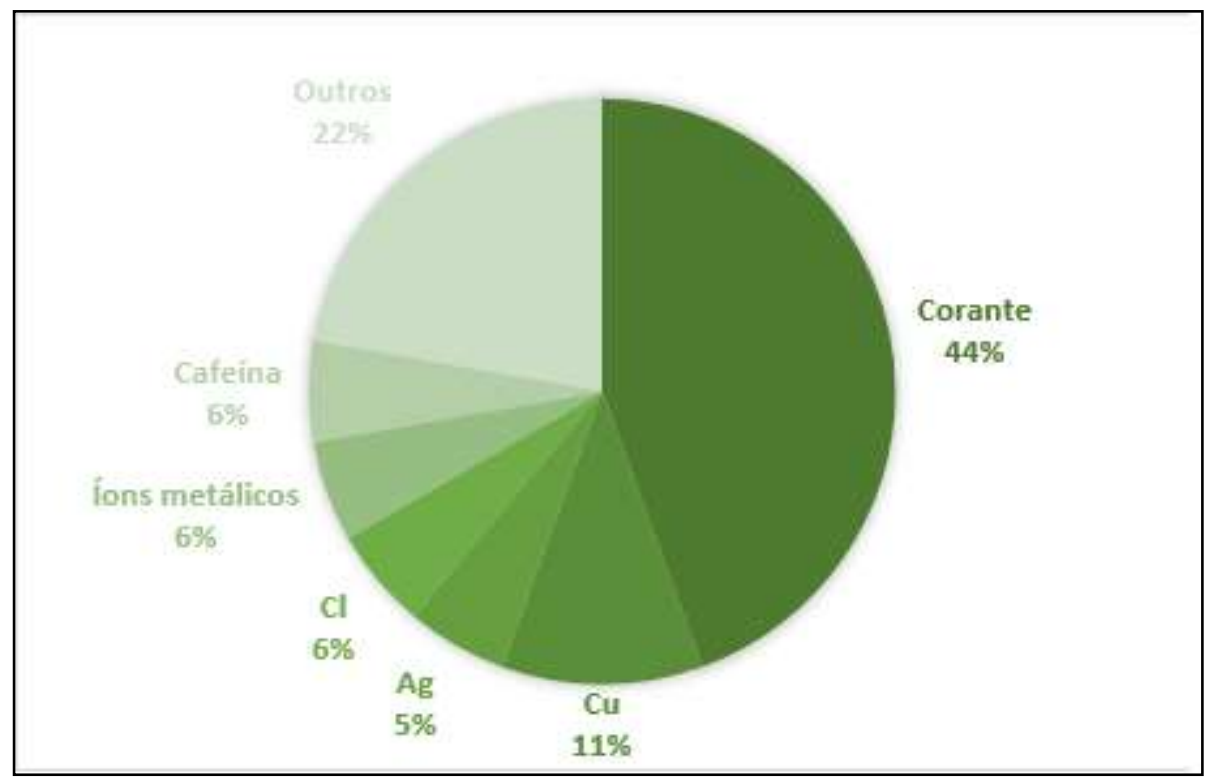

Figura 2: Materiais adsorvidos em subprodutos do babaçu. Fonte: autoria própria (2018)

Legenda: $\mathrm{Cl}$ cloro; $\mathrm{HAP}$, hidrocarbonetos aromáticos policíclicos; Ag, prata; $\mathrm{Cu}$, cobre.

Os materiais derivados do babaçu que foram estudados nessa técnica de adsorção foram: carvão ativado, epicarpo e mesocarpo (naturais e/ ou modificados) (Figura 3), com um maior número de publicações em trabalhos que utilizaram o carvão ativado produzido a partir do babaçu.

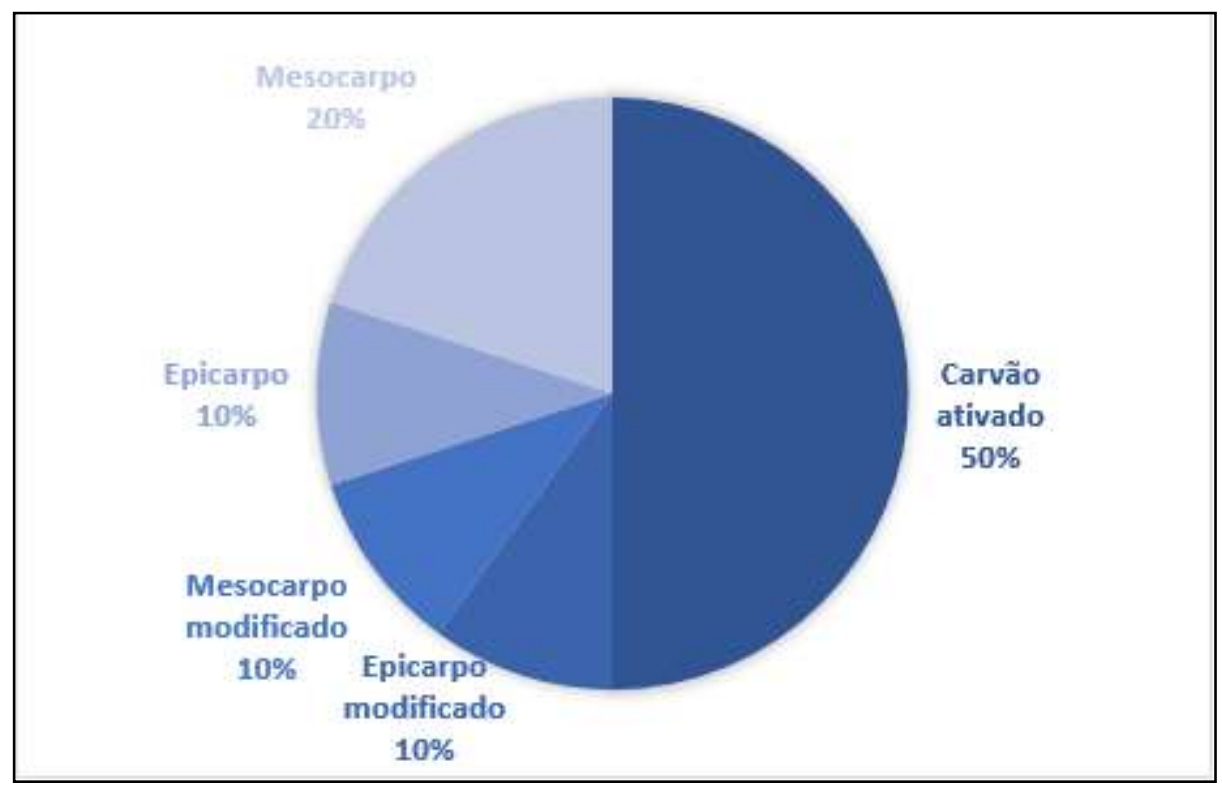

Figura 3: Porcentagem de utilização dos constituintes do coco babaçu nos estudos de descontaminação do meio ambiente. Fonte: autoria própria (2018)

\subsection{Mesocarpo}

O mesocarpo de babaçu, já é utilizado na indústria e na tecnologia há bastante tempo. Distingui-se pelo seu potencial na indústria farmacêutica devido a seu alto teor de amido, em torno de 68,3\%, biopolímero natural não tóxico e amplamente utilizado como aglutinante e desintegrante nos comprimidos e cápsulas farmacêuti- 
cas e também tem se destaca na indústria alimentícia $[9,36]$.

Vieira e colaboradores [22] experimentaram o mesocarpo como adsorvente pra os corantes Azul Remazol R160 (BR 160), Rubi S2G (R S2G), Red Remazol 5R (RR 5), Violet Remazol 5R (VR 5) e Indanthrene Olive Green (IOG), onde foram observados vários parâmetros, como tempo de agitação, $\mathrm{pH}$ e a temperatura. Onde foi mais bem ajustado o modelo cinético de pseudo-segunda ordem e o modelo capacidade de adsorção foi mais bem ajustado em Freundlich. Obtendo a seguinte ordem de adsorção nos corantes R S2G $>$ VR $5>$ BR $160>$ IOG > RR 5 .

O mesmo grupo de pesquisa ainda modificou quimicamente o mesocarpo de coco babaçu com anidrido succínico, anidrido maleico e anidrido ftálico e os utilizou para adsorver íons de cobre presentes em amostras de cachaça. Os autores concluíram que estes biopolímeros quimicamente modificados foram eficazes na adsorção dos íons cobre, portanto, sua utilização pode ser uma alternativa para enquadra as aguardentes de cana-de-açúcar às exigências da legislação brasileira no que diz respeito à contaminação por cobre [24].

Vieira e colaboradores [8] também estudaram os componentes mesocarpo e epicarpo do coco babaçu como novos biosorventes alternativos para remoção do corante têxtil ftalocianina de cobre de soluções aquosas. Os resultados mostraram que ambos os componentes são efetivos na remoção do corante têxtil Turqueza Remazol. Neste artigo é observado que o mesocarpo apresenta alterações químicas semelhantes as atribuídos a celulose, enquanto epicarpo mostra sinais característicos de lignina. Dando assim referencias mais claras sobre a composição de cada um desses. Demonstrado ainda que nessa experiência o epicarpo obteve resultados melhores no que diz respeito a adsorção, sugerindo assim a seletividade entre os adsorventes, já que o epicarpo possui um número maior de grupos funcionais e constituintes. Porem isso não fica bem claro, sendo ainda necessários estudos mais aprofundados

\subsection{Epicarpo}

O epicarpo representa $11 \%$ do fruto, é a camada mais externa, formado por fibras resistentes usadas, principalmente na fabricação de escovas, tapetes e artesanato [37]. Mas que ao mesmo tempo se mostra um bom adsorvente de corantes $[8,10]$.

Vieira e colaboradores (2011b) utilizaram o epicarpo do babaçu para remover corantes têxteis de solução aquosa. Foram estudados a adsorção de Azul Remazol R160 (BR 160), Rubi S2G (R S2G), Red Remazol 5R (RR 5), Violet Remazol 5R (VR 5) e Indanthrene Olive Green (IOG) em soluções de corante pelo epicarpo de babaçu. Os autores concluíram que a capacidade de epicarpo de babaçu em sorver os corantes apresentou-se na seguinte ordem BR 160> VR 5> RR 5> R S2G> IOG. Concluíram ainda que os dados de equilíbrio foram melhores representados pelo modelo Dubinin-Radushkevich, mostrando interações físicas entre as espécies. Esses resultados na sorção dos corantes Azul Remazol R160 (BR 160), Rubi S2G (R S2G), Red Remazol 5R (RR 5), Violet Remazol 5R (VR 5) e Indanthrene Olive Green (IOG), deu-se pela atração eletrostática entre a carga positiva epicarpo e do grupo sulfônico do corantes.

Analisando os resultados pode-se observar que alguns corantes como o (VR 5) e o (IOG), não continham em sua estrutura ligações sulfônicas, justamente esses tiveram um potencial de adsorção menor que os outros, porem cada um deles tem na sua estrutura ligações típicas de corantes, que são ligações do tipo azo, que facilitou no grau de adsorção desses corantes

Nesse artigo Vieira e colaboradores [8] mostra a similaridade entre o epicarpo e o mesocarpo e também com outros materiais lignocelulósicos, e todos são efetivos no que diz respeito a remoção do corante. Nesse artigo o modelo de pseudo-segunda ordem obteve assim como no Vieira e colaboradores [10] uma melhor adequação tanto para o epicarpo como para o mesocarpo.

Vieira e colaborados [10] mostraram que o epicarpo do coco babaçu adsorveu quase que totalmente o corante Azul Remazol R160 (BR 160), fincando assim claro a capacidade de adsorção do mesmo. Potencializando novas pesquisas, vendo quanto animadores são os resultados obtidos.

Santana e colaboradores [23] em seus estudos modificaram o mesocarpo e epicarpo de babaçu com etilenosulfito, com o intuito de adicionar centros básicos de enxofre para aumentar a atividade de coordenação de cátions divalentes presentes em soluções aquosas. Nesse estudo, os pesquisadores concluíram que o mesocarpo e epicarpo nativos apresentaram-se ineficazes quando utilizados para adsorverem íons metálicos. No entanto, após modificação química, os novos biopolímeros sintetizados, apresentaram propriedades relevantes para atuarem na remoção de cátions. Através das pesquisas de Santana e colaboradores pode-se perceber que os dois materiais antes de serem modificados não obtiveram resultados tão satisfatório, porem após esse processo apresentaram bom adsorvente de íons metálicos.

O epicarpo de babaçu também foi modificado apenas com anidrido maleico fundido sem solvente, o 
que garantiu a presença de grupo de ácidos carboxílicos na estrutura e, consequentemente, essa transformação tornou o epicarpo mais semelhante à celulose. No entanto, os grupos carboxílicos pendentes tem a capacidade de remover o cobre com o máximo de absorção através de um processo em descontínuo, melhorando o processo de adsorção [25].

\subsection{Carvão de babaçu}

Outro subproduto do babaçu que é muito utilizado é o carvão, que comumente sofre o processo de ativação. As propriedades adsortivas do carvão ativado são devido à sua estrutura porosa e a enorme área superficial. A porosidade do carvão surge devido à ao processo de oxidação do material carbonáceo. Comumente usado na purificação de óleos, clarificação de glicose, usa-se também o Carvão Ativado para remover os ingredientes que comprometeriam a pureza da bebida, dentre muitos outros [38].

Carvão ativado é um excelente adsorvente, mas o preço é alto para tratamento de água em grande escala e sua regeneração também é difícil [39]. Consequentemente, muitos pesquisadores se voltaram para os chamados adsorventes de baixo custo, tais como materiais de origem biológica, à base de minerais ou materiais alternativos para a produção de carvões [40]. Um exemplo é o carvão ativado a partir do babaçu.

Outros adsorventes (especialmente os bioadsorventes) têm certas desvantagens evidentes: as capacidades de adsorção de bioadsorventes brutos são geralmente demasiado baixas para ser usada como substitutos para carvão ativado; o número de medidas, tais como a modificação química usando $\mathrm{H}_{2} \mathrm{SO}_{4}, \mathrm{HCI}, \mathrm{HNO}_{3}$, $\mathrm{H}_{2} \mathrm{O}_{2}, \mathrm{NaOH}$ e alguns outros compostos orgânicos complexos perigosos, são aplicados para melhorar, mas estes tratamentos podem levar a efeitos colaterais muito mais grave durante a produção; mesmo após várias modificações complicadas, as capacidades de adsorção do bioadsorventes podem não ser suficientes; e os preços destes bioadsorventes após várias modificações podem não ser competitivo o suficiente [31]. Por isso, os carvões ativados ainda são os mais estudados e utilizados para adsorção de moléculas.

Os carvões ativados são adsorventes versáteis devido à sua elevada área superficial, estrutura porosa e natureza da superfície química, a qual pode ser modificada apropriadamente por tratamentos físicos e/ou químicos, visando melhorar o processo de adsorção. Esse adsorvente tem sido amplamente utilizado na remoção de diversos contaminantes presentes em efluentes [12].

Os carvões ativados do babaçu apresentaram elevada afinidade com relação ao complexo negativo de tiossulfato de prata, sendo que o carvão de coco de babaçu apresentou os melhores resultados, devido a sua maior área superficial BET (Brunauer, Emmett,Teller) em relação aos outros carvões, tornando-se um excelente adsorvente para os íons prata [12].

Jaguaribe e colaboradores [11] testaram os carvões ativados produzidos a partir de bagaço de cana, babaçu e cascas de coco para a remoção de cloro residual. Onde foi observado que o tamanho da partícula do carvão e o tempo de contado tinham influenciam na adsorção de cloro. Assim foram observados os resultados da adsorção em cada tipo de carvão através do método de BET. O carvão obtido a partir do bagaço da cana foi o único adsorvente capaz de remover $100 \%$ do cloro residual.

As cascas de coco babaçu para obtenção de carvão ativado também foram objeto de trabalho para remoção do corante azul reativo $5 \mathrm{G}$ em comparação com adsorção com óleo xisto pirolisado. Neste estudo a porosidade dos materiais foi crucial para adsorção, já que o carvão apresentou microporosidade enquanto o óleo apresentou mesoporos. Com o tamanho da molécula do corante, o óleo conseguiu adsorver de maneira mais eficaz o mesmo [13]. E que no estudo de Matta e colaboradores foi demonstrado a capacidade de adsorção de $12,9 \mathrm{mg} / \mathrm{g}$ de carvão ativado derivado das cascas de babaçu [14].

O carvão derivado do coco inteiro de babaçu ativado através de uma metodologia simples de adição de ácido e base (HPO4 e KOH) também conseguiu adsorver o corante azul de metileno em $97 \%$ após 24 horas em contato com o material. Conjuntamente com os estudos de granulometria analisados, pode-se dizer que esse carvão ativado é um forte candidato a um filtro capaz de remover resíduos [15].

A cafeína também foi removida de soluções aquosas a partir do uso de carvão ativado de babaçu [18]. O que pode deixar claro a versatilidade da utilização desse material como adsorvente de moléculas químicas.

\subsection{Outros subprodutos}

Não há estudos com a utilização de outros subprodutos do babaçu. No entanto, o endocarpo é um ótimo candidato para testes de adsorção. Já que é a parte mais fibrosa do coco babaçu, com uma maior concentração de lignina em sua estrutura e, consequentemente, com capacidade de interações inter e intra moleculares [41]. 


\section{CONCLUSÕES}

Embora ainda sejam poucos, mas os trabalhos existentes utilizando os subprodutos do babaçu como materiais adsortivos de moléculas químicas já são bastante significativos e promissores. Os estudos exibem o uso do babaçu natural, com mesocarpo e epicarpo. Assim como o uso dos subprodutos modificados, tais como mesocarpo e epicarpo após reações com anidrido succínico, anidrido maleico e anidrido ftálico, promovendo um aumento significativo de adsorção, e resultou, inclusive, na patente de um novo material adsortivo de moléculas químicas.

Outros estudos também evidenciaram a capacidade adsortiva de carvões ativados derivados do coco babaçu para adsorção de corantes e outras substâncias, no quais, quando comparados à outros carvões derivados de diferentes materiais, se mostraram inferiores em capacidade adsortiva. No entanto ainda existem muitos estudos a serem realizados, principalmente devido à produção de carvões ativados. Já que as características físico-químicas do produto podem ser alteradas, tais como a temperatura, o tempo de reação e a inserção de substâncias reativas.

Também pode-se concluir que as variáveis se adsorção são importantes em relação a remoção de moléculas químicas. $\mathrm{O}$ tamanho dos poros do material, o pH do meio, o tempo de contato e a temperatura do meio utilizados podem definir e promover um aumento no grau de adsorção desses materiais derivados do babaçu. Portanto, os subprodutos do babaçu são materiais naturais capaz de adsorver diversas moléculas químicas, podendo ser estudados também para remoção de outras classes de substâncias químicas, tais como os fármacos, pesticidas e outros contaminantes perigosos ao meio ambiente.

\section{AGRADECIMENTOS}

À Capes e à FAFEPI pelo auxílio financeiro concedido.

\section{BIBLIOGRAFIA}

[1] LEAL, G.C.G.F., FARIAS, M. S. S., ARAUJO, A. F., "O processo de industrialização e seus impactos no meio ambiente urbano", Qualitas, v. 7, n. 1, pp. 1-11, 2008.

[2] CARVALHO, J.C.B., ORSINE, J.V.C., "Contaminação do meio ambiente por fontes diversas e os agravos à saúde da população", Enciclopédia Biosfera, v. 7, n. 13, pp. 1107-1118, Nov. 2011.

[3] CRINE, G., "Non-conventional low-cost adsorbents for dye removal: A review", Bioresource Technology, v. 97, n. 9, pp. 1061-1085, Jun. 2006.

[4] FERRERO, F., "Adsorption of Methylene Blue on magnesium silicate: Kinetics, equilibria and comparison with other adsorbents", Journal of Environmental Sciences, v. 22, n. 3, pp. 467-473, Fev 2010.

[5] AHMARUZZAMAN, M., "Adsorption of phenolic compounds on low-cost adsorbents: a review", Colloid Interface Science, v. 143, n. 1-2, pp. 48-67, Nov. 2008.

[6] LORENZI, H., Palmeiras brasileiras e exóticas cultivadas, 1 ed, Nova Odessa-SP, Instituto Plantarum de Estudos de Flora, 2004.

[7] BATISTA, C.P., TORRES, O.M., MATIAS, J.E.F., et al., "Efeito do extrato aquoso de Orbignya phalerata (babaçu) na cicatrização do estômago em ratos: estudo morfológico e tensiométrico", Acta Cirurgica Brasileira, v.21, n. 3, pp. 26-32, Jan. 2006.

[8] VIEIRA, A.P., SANTANA, S.A.A., BEZERRA, C.W.B., et al., "Removal of textile dyes from aqueous solution by babassu coconut epicarp (Orbignya speciosa)", Chemical Engineering Journal, v. 173, n. 2, pp. 334-340, Set. 2011.

[9] NASCIMENTO, U.S., Carvão de Babaçu como Fonte Térmica para Sistema de Refrigeração por absorção no Estado do Maranhão, Dissertação Mestrado Profissional em Engenharia Mecânica, Universidade Estadual de Campinas, Campinas, SP, Brasil, 2004.

[10] VIEIRA, A.P., SANTANA, S.A.A., BEZERRA, C.W.B., et al., "Epicarp and Mesocarp of Babassu (Orbignya speciosa): Characterization and Application in Copper Phtalocyanine Dye Removal", Journal of the Brazilian Chemical Society, v. 22, n. 1, pp. 21-29, Jul. 2011.

[11] JAGUARIBE, E.F., MEDEIROS, L.L., BARRETO, M.C.S., et al., "The performance of activated carbons from sugarcane bagasse, babassu, and coconut shells in removing residual chlorine", Brazilian Journal of Chemical Engineering, v. 22, n. 1, pp. 41-47, Mar. 2005. 
[12] BORTOLETTO, E.C., IGARASHI-MAFRA, L., SORBO, A., et al., "Silver removal in radiographic wastewaters", Acta Scientiarum-Technology, v. 29, n. 1, pp. 37-41, Mar. 2007.

[13] LAMBRECHT, R., BARROS, M., COSSICH, E.S., et al., "Adsorption of Reactive Blue 5G dye by activated carbon and pyrolyzed shale oil residue", Adsorption Science \& Technology, v. 25, n. 10, pp. 741-749, Dez. 2007.

[14] MATTA, G.K.L., BARROS, M., LAMBRECHT, R., et al., "Dynamic Isotherms of Dye in Activated Carbon”, Materials Research-Ibero-American Journal of Materials, v. 11, n. 3, pp. 365-369, Set. 2008.

[15] CRUZ, E.T.L., DA SILVA, G.M., FREITAS, R.M., et al., "Coal active babassu adsorption in colour for the treatment of industrial waste", Periodico Tche Quimica, v. 9, n. 18, pp. 33-40, Ago, 2012.

[16] SILVA, I.D.N., ALMEIDA, J.M.F.D., FERNANDES, N.S., "Kinetic study in removal of dye in Aqueous solution by the Babassu Coconut Charcoal modified with citric acid", Periodico Tche Quimica, v. 14, n. 28, pp. 42-49, Jan. 2017.

[17] PALMA, L.S.O., BORGES, S.L.F., ITAÚNA S.P.Á., et al., "A proposal for a trial lesson using babassu mesocarp (orbignya speciosa) in the removal of methylene blue from aqueous solutions", Educacion Quimica, v. 26, n. 4, pp. 314-318, Ago. 2015.

[18] COUTO, O.M., MATOS, I., DA FONSECA, I.M., et al., "Effect of solution pH and influence of water hardness on caffeine adsorption onto activated carbons", Canadian Journal of Chemical Engineering, v. 93, n. 1, pp. 68-77, Nov. 2015.

[19] ALVES, M.R.R., ZUNIIGA, A.D.G., SOUSA, R.D.C.S., et al., "The Process of Separating Bovine Serum Albumin Using Hydroxyapatite and Active Babassu Coal (Orbignya martiana)", Scientific World Journal, v.2016, pp. 1-9, Jul. 2016.

[20] PAIXAO, R.M., RECK, I.M., BERGAMASCO, R., et al., "Activated carbon of Babassu coconut impregnated with copper nanoparticles by green synthesis for the removal of nitrate in aqueous solution", Environmental technology, v. 4, pp. 1-10, Jul. 2017.

[21] SCHMITT, C.C., CHIARO, S.S.X., TANOBE, V.O.D.A., et al., "Regeneration of activated carbon from babassu coconut refuse, applied as a complementary treatment to conventional refinery hydrotreatment of diesel fuel", Journal of Cleaner Production, v. 40, n. 3, pp. 1465-1469, Jan. 2017.

[22] VIEIRA, A.P., SANTAN, S.A., BEZERRA, C.W., et al., "Kinetics and thermodynamics of textile dye adsorption from aqueous solutions using babassu coconut mesocarp", Journal of Hazardous Materials, v. 166, n. 2-3, pp. 1272-1278, Jul. 2009.

[23] SANTANA, S.A.A., VIEIRA, A.P., SILVA, E.C., et al., "Immobilization of ethylenesulfide on babassu coconut epicarp and mesocarp for divalent cation sorption", Journal of Hazardous Materials, v. 174, n. 1-3, pp. 714-719, Feb. 2010.

[24] VIEIRA, A.P., SANTANA, S.A.A., BEZERRA, C.W.B., et al., "Copper sorption from aqueous solutions and sugar cane spirits by chemically modified babassu coconut (Orbignya speciosa) mesocarp", Chemical Engineering Journal, v. 161, n. 1-2, pp. 99-105, Jul. 2010.

[25] VIEIRA, A.P., SANTANA, S.A.A., BEZERRA, C.W.B., et al., "High performance maleated lignocellulose epicarp fibers for copper ion removal”, Brazilian Journal of Chemical Engineering, v. 31, n. 1, pp. 183193, Mar. 2014.

[26] GOMES, M., NASCIMENTO, J.R., SILVA, M.G.O., "Mesocarp babassu (Orbinya sp) as adsorbent dodecyl benzene sodium sulfonate (sdbs)", Holos, v. 32, n. 7, pp. 51-61, Sep. 2016.

[27] MOTOO, H., inventor COSMETIC COMPOSITION1987.

[28] VIEIRA, A.P., AIROLDI, C., BEZERRA, C.W., et al., "Processo de preparação de mesocarpo do coco de babaçu quimicamente modificado; Mesocarpo de babaçu quimicamente modificado, processo de remoção de íons metálicos em soluções hidroetanólicas e uso do mesocarpo do coco de babaçu quimicamente modificado". PI 1004478-7 A2. 2010.

[29] FRANCO, F.J.P., Utilização da fibra do epicarpo do coco babaçu em compósito com matriz de epóxi: Estudo do efeito do tratamento da fibra. Dissertação de M.Sc., Programa de pós-graduação em ciências e engenharia dos materiais, Universidade Federal do Rio Grande do Norte, Natal, RN, Brasil, 2010.

[30] MELLO, V.F.B., GAMA, J., FERREIRA, J.M., et al., "Estudo e caracterização de propriedades da argila esmectita na remoção de corantes têxteis", Matéria,v. 18, pp. 1467-1482, Dec. 2013.

[31] FENG, Y., DIONYSIOU, D.D., WU, Y., et al., "Adsorption of dyestuff from aqueous solutions through 
oxalic acid-modified swede rape straw: Adsorption process and disposal methodology of depleted bioadsorbents", Bioresource Technology, v. 138, pp. 191-197, Jun. 2013.

[32] SILVA, L.S., LIMA, L.C.B., SILVA, F.C., et al., "Dye anionic sorption in aqueous solution onto a cellulose surface chemically modified with aminoethanethiol", Chemical Engineering Journal, v. 2018, pp. 8998, Feb. 2013.

[33] ALVARENGA, F.W., MARTELLI, P.B., GORGULHO, H.F., "Carvões ativados utilizando palha de milho como matéria prima, obtenção e uso". PI0804435-0 A2. 2010.

[34] MELO, J.C.P., Síntese e caracterização de derivados da celulose modificada com anidridos orgânicosadsorção e termodinâmica de interação com cátions metálicos, Dissertação de M.Sc., UNICAMP, Instituto de química, Campinas, São Paulo, Brasil, 2007.

[35] BRAGATO, M., EL SEOUD, O.A., "Formation, properties, and "ex situ" soil decontamination by vegetable oil-based microemulsions", Journal of Surfactants and Detergents, v. 6, n. 3, pp. 143-150, Abr. 2003.

[36] DERMIRGOZ, D., "Chemical modification of starch based biodegradable polymeric blends: effects on water uptake, degradation behavior", Polymer Degradation Stability, v.70, n. 2, pp. 161-170, Dec. 2000.

[37] SILVA, A.P.S., Caracterização físico-química e toxicológica do pó de mesocarpo do babaçu (Orbignya phalerata Mart): subsídio para o desenvolvimento de produtos, Dissertação de M.Sc., Programa de pósgraduação em ciências farmacêuticas, UFPI, Piauí, Brasil, 2011.

[38] MUCCIATO, J.C., "Conceitos e aplicações do carbono ativado", Revista meio filtrante, v. 5, pp. 1-9, Out. 2015.

[39] GUPTA, V.K., ALI, I., SALEH, T., et al., "Chromium removal from water by activated carbon developed from waste rubber tires", Environmental Science and Pollution Research, v. 20, n. 3, pp. 1261-1268, Mar, 2013.

[40] MITTER, C., DULLER, C., FELDBOUER-DURSTMÜLER, B., et al., "Internationalization of family firms: the effect of ownership and governance", Review of Managerial Science, v. 6, n.4, pp. 1-28, Set. 2012.

[41] TEIXEIRA, M.A., "Biomassa de babaçu no Brasil", In: Encontro De Energia No Meio Rural, pp. 1-10, Campinas, São Paulo, 2002.

\section{ORCID}

Josany Saibrosa da Silva

Mirian Lima dos Santos

Edson Cavalcante da Silva Filho

Maria das Graças Freire de Medeiros Carvalho

Livio Cesar Cunha Nunes https://orcid.org/0000-0002-2806-6381

https://orcid.org/0000-0002-8817-1212

https://orcid.org/0000-0003-0988-2970

https://orcid.org/0000-0002-6161-7085

https://orcid.org/0000-0002-1178-7940 